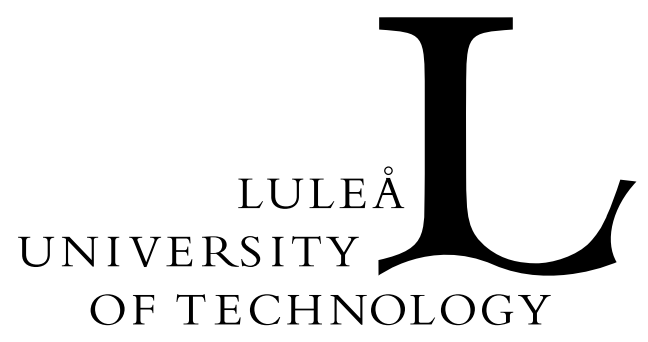

Division of Signal Processing

Visiting address: Universitetsområdet, Porsön, Luleå Postal address: SE-971 87, Luleå, Sweden

Telephone: +46920910 00. Fax: +4692072043

URL: http://www.sm.luth.se/csee/sp/

\title{
Estimation of the Spatial Impulse Response of an Ultrasonic Transducer Using a Tomographic Approach
}

\author{
Magnus Sandell and Anders Grennberg
}

In Journal of the Acoustical Society of America, vol. 98, no. 4, pp. 2094-2103, October 1995.

(C) 1995 Acoustical Society of America 


\title{
Estimation of the spatial impulse response of an ultrasonic transducer using a tomographic approach
}

\author{
Magnus Sandel| ${ }^{\mathrm{a})}$ and Anders Grennberg \\ Division of Signal Processing, Luleå University of Technology, 97187 Luleå, Sweden
}

(Received 23 December 1994; accepted for publication 2 June 1995)

\begin{abstract}
The impulse response method is often used to study the ultrasonic field radiated by planar or gently curved transducers. These investigations can be extended to include the reflected echoes from point reflectors, which can be used in synthesizing echoes from reflectors of known shapes. The single point echo can be measured directly but there are however certain problems associated with this. This paper proposes an alternative method to estimate the echo from a pointlike reflector by measuring the echoes from sliding half-planes. Using a tomographic approach, the single point echo is estimated from these measurements. To perform the tomographic inversion, a truncated weighted singular value decomposition (SVD) is used in order to catch essential features of the ultrasonic echoes, where the weight functions are chosen especially to suit this application. Numerical solutions are given for the chosen weight functions and an error analysis is carried out. (C) 1995 Acoustical Society of America.
\end{abstract}

PACS numbers: $43.60 . P t, 43.20 . F n, 43.38 .-p$

\section{INTRODUCTION}

The impulse response method is often used to study the ultrasonic field radiated by planar or gently curved transduccrs. ${ }^{1-3}$ Thesc investigations can be extended to include the reflected echo from a pointlike reflector placed in the ultrasonic field. These echoes can be useful in predicting the echoes from reflectors of known shapes. ${ }^{4}$ They may also be of use in improving the pulse-echo imaging method. This method measures the distance to an object by estimating the time-of-flight (TOF), i.e., the time between transmission of an ultrasonic pulse and the arrival of the echo. ${ }^{5,6}$ This can be done in several ways which are described in various articles in Refs. 7 and 8 . However, this assumes that the two echoes have the same signal shape. Any differences in the shapes will degrade the TOF estimate. This might, however, be used to extract more information in the echoes apart from the arrival time, such as the slope of the surface at the measured point. Two echoes from a plane surface with a sloping angle of $0^{\circ}$ and $8^{\circ}$, respectively, are shown in Fig. 1, illustrating the fact that the shape of the echo is dependent on the angle of inclination. ${ }^{9}$

When ultrasound is used for measurements in air, a focused transducer is usually used due to the high attenuation. If the reflecting object is kept close to the focal plane of the transducer, the reflecting echo will originate from a very small area on the object. This might be modeled as a plane, sloping surface. Apart from the TOF estimate, which gives the axial distance to the object, an estimate of the slope may also be obtained. If this estimate is good, a sparser scanning grid may be used while maintaining the same accuracy of the estimated surface profile.

By neglecting nonlinear effects in the medium and the transducer, the ultrasonic echo from a pointlike reflector may be modeled as a linear system, ${ }^{10}$ see Fig. 2 . The transducer is assumed to be circular symmetric and uniformly excited.

\footnotetext{
a)Electronic mail: mangus@sm.luth.se
}

From the reciprocity property, ${ }^{11}$ we may put the transfer functions in transmission and reception modes equal, i.e. $T_{T}(t)=T_{R}(t)=T(t)$ and $h_{T}(\bar{r}, t)=h_{R}(\bar{r}, t)=h(\bar{r}, t)$. The spatial impulse response $h(\bar{r}, t)$ of the transducer has been calculated analytically for a focused transducer in Refs. 1-3. The electromechanical transfer function $T(t)$ is however difficult to calculate and must be estimated from measurements. At the focal point of the transducer, the spatial impulse response is $h(\overline{0}, t)=\delta(t-R / c)$, where $\delta(\cdot)$ is the Dirac delta function, $R$ is the curvature of the transducer, and $c$ is the speed of sound in the medium.

Since all estimated point echoes depend on the focal point echo, it is essential that the latter has high accuracy. To measure the focal point echo directly is difficult. An object would have to be very small to be considered pointlike and this would result in a weak echo and consequently a low SNR. Increasing the size will result in spatial smoothing and deconvolution is necessary. To avoid these practical limitations, we propose an indirect method instead by using a tomographic approach. ${ }^{12}$ This method gives us an independent estimation of all single point echoes in the field simultaneously. In tomography, the line integral of a twodimensional function is normally measured. Methods for inverting these measurements to the original function are known, at least in theory. ${ }^{13}$ In practice, restrictions like a finite number of projections limit the accuracy of these inversions. ${ }^{14}$ However, we chose instead to measure the echoes from half-planes, i.e., the integrals of the line echoes. The reason for this is a better SNR and to avoid the spatial smoothing that will occur from a line reflector since it cannot be made infinitesimal small.

\section{THEORY}

\section{A. Single-point echoes}

In our experiments we have assumed that our focused transducer is axisymmetric. The assumption of symmetry is for reasons of simplicity, but the method can be extended to 


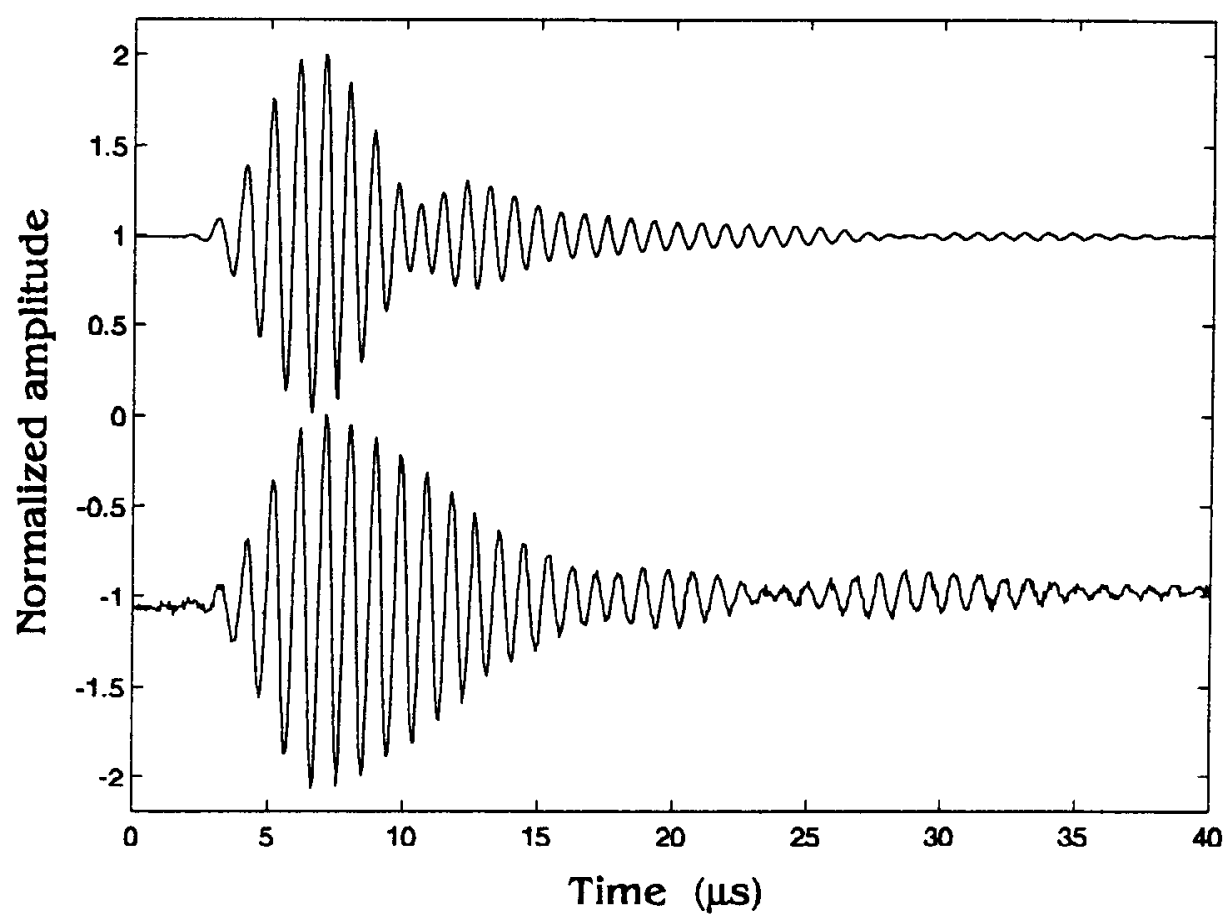

FIG. 1. Echoes from plane surfaces with normalized amplitudes. Perpendicular (above) and $8^{\circ}$ angle of inclination (below).

unsymmetric transducers. Suppose that we have a plane that is parallel to the focal plane and is located at a height $z$ from the transducer. The plane has a perpendicular distance $p$ to the axis of the transducer. The transducer works both as a transmitter and a receiver. The echo $g$ from the line will be a function of distance $p$, height $z$, and time $t$. Using superposition we can express the echo from a plane in terms of the single point echoes. Since the height $z$ was constant in our experiment and $t$ is only a parameter in our calculations, we will drop them from now on.

Let $f(r)$ denote the echo from a single point at a distance $r$ from the axis of symmetry. Since the transducer is focused, the reflection from a point far away from the axis of symmetry can be neglected. This means that we can put $f(r)=0$ for $|r|>1$ in a normalized scale. What the spatial distance $r=1$ corresponds to depends on the geometry of the transducer and how accurate the approximation is. The echo $g(p)$ from a plane $P$ with a perpendicular distance $p$ to the axis of symmetry, will then be the integral of $f(r)$ over $P$,

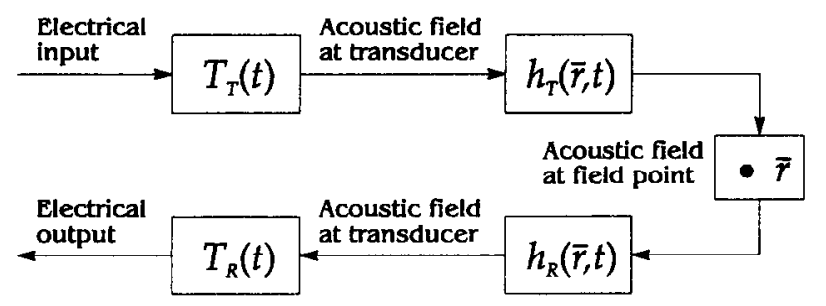

FIG. 2. Ultrasonic echo modeled as a linear system.

$$
g(p)=\int_{P} f(r) d s=\int_{0}^{1} K(p, r) f(r) d r,
$$

where the integration area $P$ is defined in Fig. 3.

The integration kernel $K(p, r)$ is derived in Appendix A and is found to be

$$
K(p, r)=\left\{\begin{array}{l}
2 \pi r, \quad-1 \leqslant p<-r \\
2 r \arccos (p / r), \quad-r \leqslant p<r, \\
0, \quad r \leqslant p \leqslant 1 .
\end{array}\right.
$$

The transducer is axisymmetric, so we may assume the plane to be parallel to the $y$ axis for the sake of simplicity. As can be seen from Eq. (1), we have a mapping from the single point echoes $f(r)$ to the half-plane echoes $g(p)$ via the integral kernel. Since the projections are along planes and not

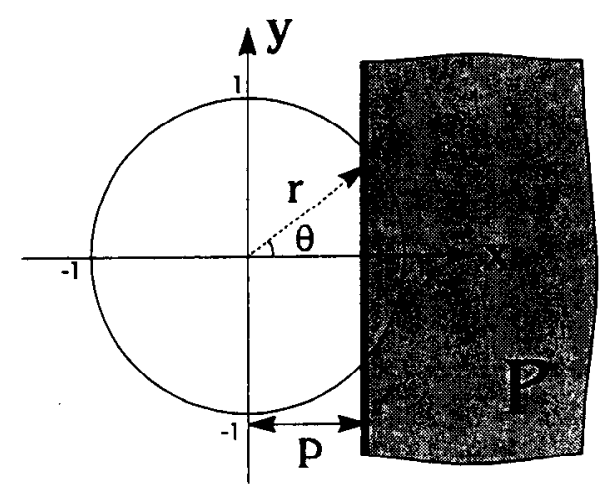

FIG. 3. Integration area from reflecting half-plane in the focal plane. 


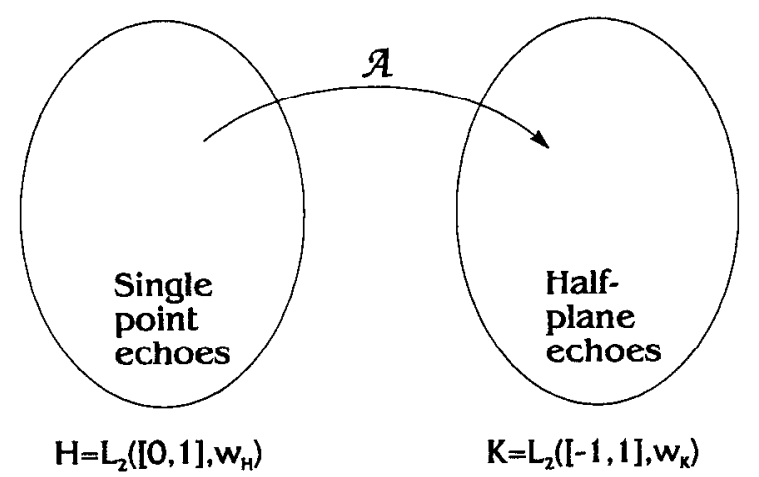

FIG. 4. Half-plane echoes viewed as a mapping between Hilbert spaces.

lines, this is a modified version of tomography. Using some techniques from that area, an inversion formula for the single point echoes can be constructed. There is some a priori knowledge of $f(r)$ which should be used. The weighted Hilbert spaces $H$ and $K$ is, respectively, defined as $H=L_{2}\left([0,1], w_{H}\right)$, which contain the single point echoes $f(r)$, and $K=L_{2}\left([-1,1], w_{K}\right)$, which contains the halfplane echoes $g(p)$. The scalar product of these spaces are defined as

$$
\begin{aligned}
& \left(f_{1}, f_{2}\right)_{H}=\int_{0}^{1} f_{1}(r) f_{2}(r) w_{H}(r) d r, \\
& \left(g_{1}, g_{2}\right)_{K}=\int_{-1}^{1} g_{1}(p) g_{2}(p) w_{K}(p) d p .
\end{aligned}
$$

The weight functions $w_{H}(r)$ and $w_{K}(p)$ will be discussed in Sec. I D. Thus, with the kernel in Eq. (1), an integral operator $A$ is defined, mapping the single point echoes $f(r) \in H$ to the half-plane echoes $g(p) \in K$, see Fig. 4:

$$
\begin{aligned}
& g=A f f \\
& \not B: H \rightarrow K .
\end{aligned}
$$

\section{B. Inversion by regularization}

In order to find the single point echoes, one must find the inverse of the operator $A$ of Eq. (5). This equation is however ill-posed, since $\mathscr{A}^{-1}$ is not continuous, i.e., the solution $f$ does not continuously depend on $g .{ }^{14}$ This will cause instability since a small change in $g$ will result in a large error in $f$. The goal is to approximate the solution $f$ from a knowledge of a perturbed $g^{\delta}$ with a known error level, $\left\|g^{\delta}-g\right\|_{K} \leqslant \delta$. When $g$ belongs to the range $R(H)$ $=\{\mathscr{L} f: f \in H\}$ then there exists a solution $f$ of $\mathscr{b} f=g$. For a perturbed right-hand side, we cannot expect $g^{\delta} \in R(H)$ in general. Using the erroneous data $g^{\delta}$, a reasonable approximation $f^{\delta}$ to the exact solution $f$ of the unperturbed equation $\not f f=g$ is sought. Of course, this approximation should be stable, i.e., $f^{\delta}$ depends continuously on the actual data $g^{\delta}$. Therefore we introduce the approximation of the unbounded inverse operator $f^{-1}: R(H) \rightarrow H$ by a bounded linear opera- tor $\mathscr{B}_{\alpha}: K \rightarrow H$. A family of operators $\mathscr{B}_{\alpha}$, with the regularization parameter $\alpha>0$, is called a regularization scheme ${ }^{15}$ for the operator $\mathscr{A}$ if

$$
\lim _{\alpha \rightarrow \infty} \mathscr{B}_{\alpha} \mathscr{B} f=f, \quad \forall f \in H .
$$

The regularization scheme approximates the solution $f$ of $\not \partial f-g$ by the regularized solution

$$
f_{\alpha}^{\delta}=\mathscr{R}_{\alpha} g^{\delta} \text {. }
$$

\section{Singular value decomposition}

A regularization scheme $\mathscr{B}_{\alpha}$ can be constructed by using the singular value decomposition (SVD) of the operator $\mathscr{A}$. From the SVD, two orthonormal systems $\left\{f_{n}\right\},\left\{g_{n}\right\}$ for $H$, $K$, respectively, are constructed. ${ }^{15}$ These two systems are related by

$$
\begin{aligned}
& \not A f_{n}=\sigma_{n} g_{n}, \\
& \not A^{*} g_{n}=\sigma_{n} f_{n},
\end{aligned}
$$

where $\mathscr{A}^{*}$ is the adjoint operator of $\mathscr{t}$ and $\sigma_{n}$ are singular values of $\mathscr{B}$, which are assumed to be ordered, i.e., $\sigma_{1} \geqslant \sigma_{2} \geqslant \ldots$. The adjoint operator . $\mathscr{A}^{*}$ is defined from $\left(f, \mathscr{A}^{*} g\right)_{H}=(\mathscr{f} f, g)_{K}, \forall f \in H, \forall g \in K$. It can be shown ${ }^{15}$ that if $g=\Sigma_{n} a_{n} g_{n}$, then the spectral cutoff,

$$
\mathscr{R}_{N} g=\sum_{n=1}^{N} \frac{1}{\sigma_{n}} a_{n} f_{n}
$$

describes a regularization scheme with parameter $N$ and $\left\|\mathscr{R}_{N}\right\|=1 / \sigma_{N}$.

\section{Weighted SVD}

By studying the Radon transform, ${ }^{14}$ suitable weight functions can be derived. The Radon transform maps realvalued functions in $\mathbb{R}^{N}$ to their integrals over $(N-1)$ dimensional hyperplanes. For $N=2$ this means line integrals and in our case the Radon transform corresponds to the mapping between the point echoes to the line echoes. In Ref. 16 a complete singular system for the Radon transform, as a mapping between weighted Hilbert spaces, has been calculated. Assuming circular symmetry, the Radon transform reduces to

$$
\mathscr{R}: L_{2}\left([0,1], U_{\nu}^{-1}\right) \rightarrow L_{2}\left([-1,1], V_{\nu}^{-1}\right),
$$

where the weight functions are

$$
\begin{aligned}
& U_{\nu}(r)=r\left(1-r^{2}\right)^{\nu-1}, \\
& V_{\nu}(p)=\left(1-p^{2}\right)^{\nu-1 / 2} .
\end{aligned}
$$

Note that an extra $r$ is introduced in $U_{\nu}(r)$ compared to Ref. 16 since a change from Cartesian to polar coordinates is implied. The parameter $\nu$ is used to determine if the weight should be large at the boundary $(\nu>1)$ or at the center $(\nu<1)$. From the nature of the echoes, we know that their amplitude decreases as the distance from the axis of symmetry increases. By putting a large weight at the boundary, we can create Hilbert spaces whose functions will decay sufficiently fast (otherwise their norm would not be finite, i.e., they 
would not belong to the Hilbert space). In this way we can construct two orthonormal bases whose base functions have the same shape as the echoes. From the measurement, the decay of the echoes can be estimated and appropriate weight functions designed. Since our operator $\mathscr{A}$ is the integral of the Radon transform $\mathscr{B}$, we define the weight function for the half-plane echo space $K$ as $W_{\nu}(p)=\int_{p}^{1} V_{\nu}(x) d x$, which results in the half-plane echo space

$$
K=L_{2}\left([-1,1], W_{\nu}^{-1}\right) \text {. }
$$

The choice of $\nu$ must be such that $f \in H \Rightarrow \mathscr{A f} \in K$. If the half-plane echoes decay as $(1-p)^{\epsilon}$ when $p \rightarrow 1$, it is sufficient that $\nu<2 \epsilon-1 .{ }^{17}$ For the transducer used in the experiment, see Sec. III, we found $\epsilon \approx 3$ and we made the choice $\nu=4$.

To summarize, we define the operator $t b$ which maps the point echoes $f(r)$, which belong to the Hilbert space $H$, to the half-plane echoes $g(p)$ in Hilbert space $K$ :

$$
\begin{aligned}
& g=\mathscr{f f}, \\
& \mathscr{f}: L_{2}\left([0,1], U_{4}{ }^{1}\right) \rightarrow L_{2}\left([-1,1], W_{4}{ }^{1}\right),
\end{aligned}
$$

where

$$
\begin{aligned}
U_{4}(r)= & r\left(1-r^{2}\right)^{3}, \\
W_{4}(p)= & \int_{p}^{1} V_{4}(x) d x \\
= & \frac{35}{128} \arccos (p)-\frac{p}{384} \sqrt{1-p^{2}} \\
& \times\left(279-326 p^{2}+200 p^{4}-48 p^{6}\right) .
\end{aligned}
$$

\section{ERROR ESTIMATES}

\section{A. Error model}

As was found in Sec. II, the regularization scheme approximates the solution $f$ of $\mathscr{A} f=g$ by the regularized solution $f_{\alpha}^{\delta}=\mathscr{B}_{\alpha} g^{\delta}$. By writing $f_{\alpha}^{\delta}-f=\mathscr{R}_{\alpha} g^{\delta}-\mathscr{B}_{\alpha} g$ $+\mathscr{B}_{\alpha} b f-f$, using the triangle inequality and $\left\|g^{\delta}-g\right\|_{K} \leqslant \delta$, an estimate of the error can be written as

$$
\left\|f_{\alpha}^{\delta}-f\right\|_{H} \leqslant \delta\left\|\mathscr{B}_{\alpha}\right\|+\left\|\mathscr{B}_{\alpha} \not b f-f\right\|_{H} .
$$

This decomposition illustrates the fact that we have two types of error: the first term reflects mainly the influence of the incorrect data and the second term is due to the approximation error between $\mathscr{B}_{\alpha}$ and $\mathscr{A}^{-1}$. The latter will decrease as $\alpha$ increases but the norm $\left\|\mathscr{R}_{\alpha}\right\|$ will increase unbounded since $\mathscr{R}_{\alpha} \rightarrow \mathscr{A}^{-1}$ as $\alpha \rightarrow \infty$ and $\mathscr{C}^{-1}$ is unbounded. Hence, the choice of $\alpha$ will be a trade-off between approximation error and data error.

\section{B. Data error}

Using the spectral cut-off method, the measured echo $g^{\delta}$ is decomposed using $N$ terms, i.e.,

$$
g^{N}=\sum_{n=1}^{N} a_{n} g_{n} .
$$

Assume that the data error consists of additive zero-mean white Gaussian noise $u(p)$ with variance $\delta^{2}$. Since $u(p)$ cannot be expected to belong to $K$, the measured echo $g^{\delta}=g+u$ does not lie in $K$. In order to minimize the effect of the noise, the coefficients $a_{n}$ should be chosen so that the residual $g^{\delta}-g^{N}$ is minimized in the Euclidian norm. The orthogonality principle states that $a_{n}$ should be chosen such that

$$
\left(g^{\delta}-\sum_{n=1}^{N} a_{n} g_{n}, g_{k}\right)_{2}=0, \quad k=1,2, \ldots, N .
$$

This gives us the following system of equations:

$$
\begin{aligned}
\mathbf{G a} & =\left(\begin{array}{ccc}
\left(g_{1}, g_{1}\right)_{2} & \cdots & \left(g_{1}, g_{N}\right)_{2} \\
\vdots & \ddots & \vdots \\
\left(g_{N}, g_{1}\right)_{2} & \cdots & \left(g_{N}, g_{N}\right)_{2}
\end{array}\right)\left(\begin{array}{c}
a_{1} \\
\vdots \\
a_{N}
\end{array}\right) \\
& =\left(\begin{array}{c}
\left(g^{\delta}, g_{1}\right)_{2} \\
\vdots \\
\left(g^{\delta}, g_{N}\right)_{2}
\end{array}\right)=\left(\begin{array}{c}
\left(g, g_{1}\right)_{2} \\
\vdots \\
\left(g, g_{N}\right)_{2}
\end{array}\right)+\left(\begin{array}{c}
\left(u, g_{1}\right)_{2} \\
\vdots \\
\left(u, g_{N}\right)_{2}
\end{array}\right) \\
& =\mathbf{g}+\mathbf{u} .
\end{aligned}
$$

From $\mathbf{G a}=\mathbf{g}+\mathbf{u}$, implying $\mathbf{a}=\mathbf{G}^{-1} \mathbf{g}+\mathbf{G}^{-1} \mathbf{u}$, the covariance matrix for $\mathbf{a}$ is found to be

$$
\begin{aligned}
\mathbf{R}_{a a} & =E\left[(\mathbf{a}-E[\mathbf{a}])(\mathbf{a}-E[\mathbf{a}])^{T}\right]=E\left[\mathbf{G}^{-1} \mathbf{u u}^{T} \mathbf{G}^{-1}\right] \\
& =\mathbf{G}^{-1} \mathbf{R}_{u u} \mathbf{G}^{-1} .
\end{aligned}
$$

The term $r_{i j}$ in the covariance matrix $\mathbf{R}_{u u}=E\left[\mathbf{u u}^{T}\right]$ is

$$
\begin{aligned}
r_{i j} & =E\left[\left(u, g_{i}\right)_{2}\left(u, g_{j}\right)_{2}\right] \\
& =E\left(\int_{-1}^{1} u(p) g_{i}(p) d p \int_{-1}^{1} u(x) g_{j}(x) d x\right) \\
& =\int_{-1}^{1} \delta^{2} g_{i}(p) g_{j}(p) d p=\delta^{2}\left(g_{i}, g_{j}\right)_{2} .
\end{aligned}
$$

This means that $\mathbf{R}_{u u}=\delta^{2} \mathbf{G}$ and that $\mathbf{R}_{a a}=\delta^{2} \mathbf{G}^{-1}$. The reconstructed single point echo is

$$
\begin{aligned}
f^{N} & =\sum_{n=1}^{N} \frac{1}{\sigma_{n}} a_{n} f_{n}=\left[f_{1} \cdots f_{N}\right] \Sigma^{-1}\left[a_{1} \cdots a_{N}\right]^{T} \\
& =\mathbf{f}^{T} \mathbf{\Sigma}^{-1} \mathbf{a},
\end{aligned}
$$

where $\Sigma^{-1}=\operatorname{diag}\left(\sigma_{1}^{-1}, \ldots, \sigma_{N}^{-1}\right)$. It should be noted here that $f^{N}$ is not really a scalar but a function of radius $r$. The expectance and variance of the reconstructed single point echo $f^{N}$ are

$$
\begin{aligned}
E\left[f^{N}\right] & =E\left[\mathbf{f}^{T} \mathbf{\Sigma}^{-1} \mathbf{a}\right]=\mathbf{f}^{T} \mathbf{\Sigma}^{-1} E[\mathbf{a}]=\mathbf{f}^{T} \mathbf{\Sigma}^{-1} \mathbf{G}^{-1} \mathbf{g}, \\
\operatorname{Var}\left[f^{N}\right] & =E\left[\left(f^{N}-E\left[f^{N}\right]\right)^{2}\right]=E\left[\left(\mathbf{f}^{T} \mathbf{\Sigma}^{-1}\left(\mathbf{a}-\mathbf{G}^{-1} \mathbf{g}\right)\right)^{2}\right] \\
& =E\left[\left(\mathbf{f}^{T} \mathbf{\Sigma}^{-1} \mathbf{G}^{-1} \mathbf{u}\right)\left(\mathbf{f}^{T} \mathbf{\Sigma}^{-1} \mathbf{G}^{-1} \mathbf{u}\right)^{T}\right] \\
& =E\left[\mathbf{f}^{T} \boldsymbol{\Sigma}^{-1} \mathbf{G}^{-1} \mathbf{u} \mathbf{u}^{T} \mathbf{G}^{-T} \mathbf{\Sigma}^{-T} \mathbf{f}\right] \\
& =\mathbf{f}^{T} \mathbf{\Sigma}^{-1} \mathbf{G}^{-1} \mathbf{R}_{u u} \mathbf{G}^{-1} \mathbf{\Sigma}^{-1} \mathbf{f} \\
& =\delta^{2} \mathbf{f}^{T} \mathbf{\Sigma}^{-1} \mathbf{G}^{-1} \mathbf{\Sigma}^{-1} \mathbf{f} .
\end{aligned}
$$

The variance can be estimated by 


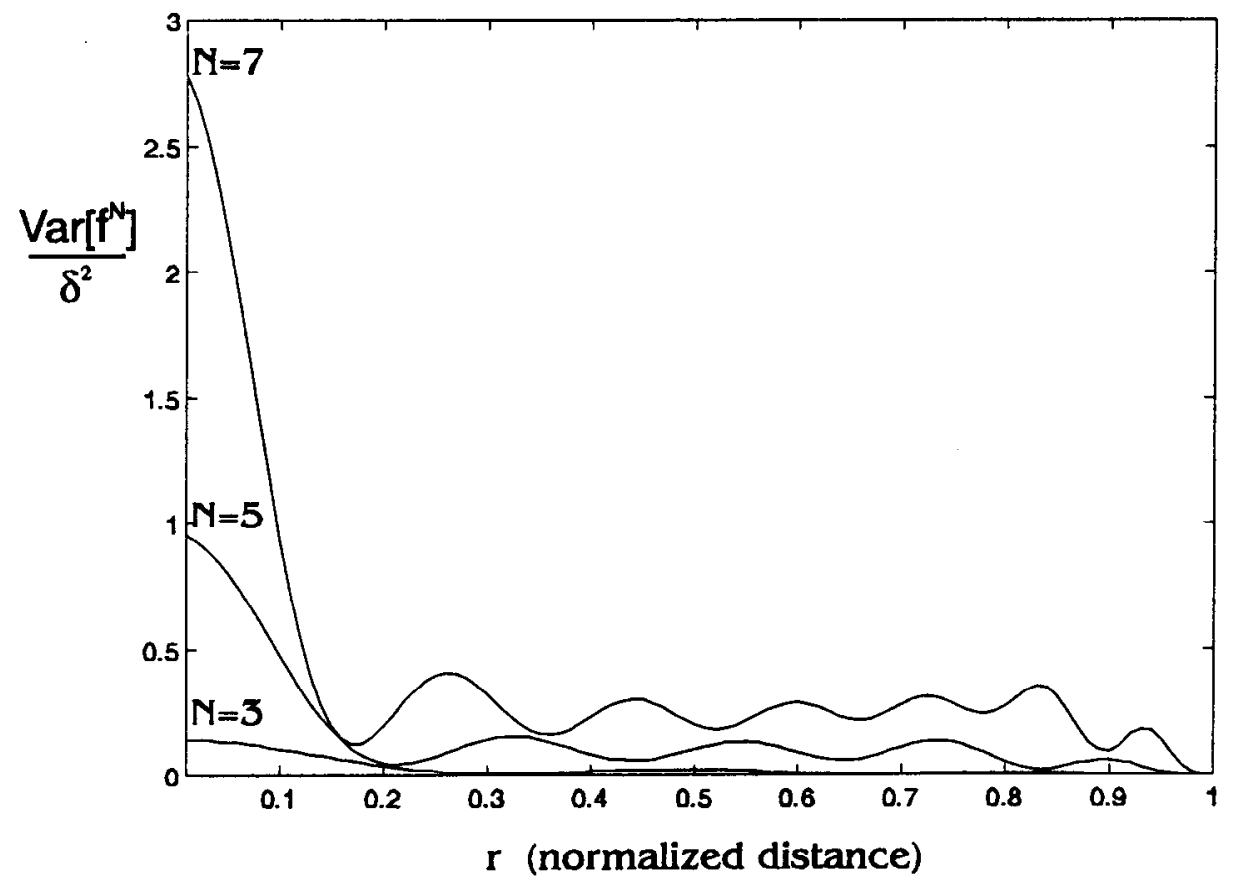

FIG. 5. Error variance $R_{f f}(r)$ for different values of $N$, the number of terms in the spectral cutoff.

$$
\begin{aligned}
\operatorname{Var}\left[f^{N}\right] & =\delta^{2}\left(\Sigma^{-1} \mathbf{f}\right)^{T} \mathbf{G}^{-1}\left(\Sigma^{-1} \mathbf{f}\right) \leqslant \frac{\delta^{2}}{\lambda_{\min }}\left\|\mathbf{\Sigma}^{-1} \mathbf{f}\right\|^{2} \\
& \leqslant \frac{\delta^{2}}{\lambda_{\min } \sigma_{N}^{2}}\|\mathbf{f}\|^{2}
\end{aligned}
$$

where $\lambda_{\min }$ is the smallest eigenvalue of $\mathbf{G}$. Since $\sigma_{N}$ decreases for increasing $N$ we can expect variance of $f^{N}$ to grow for larger $N$. In Fig. 5, the variance for $N=3,5,7$ is plotted.

The variance also depends on the smallest eigenvalue $\lambda_{\min }$ of $\mathbf{G}$. For the standard scalar product, i.e., the weight function $w_{K}(p)=1$, it is one since $\mathbf{G}$ becomes the identity matrix. The more weight one puts on the functions as $p \rightarrow 1$, the less diagonal the matrix becomes and thus the smallest eigenvalue decreases.

\section{Approximation error}

The second part of Eq. (17) is the approximation error. To analyze the effect of using a regularized inverse, the data error is ignored and only the approximation error is treated. The regularization parameter is $N$, the number of terms in the spectral cutoff. By using

$$
\left(f, f_{n}\right)_{H}=\frac{1}{\sigma_{n}}\left(f, \mathscr{A}^{*} g_{n}\right)_{H}=\frac{1}{\sigma_{n}}\left(\mathscr{A f}, g_{n}\right)_{K}=\frac{1}{\sigma_{n}}\left(g, g_{n}\right)_{K}
$$

the error due to $\mathscr{R}_{N}$ can be written as

$$
\begin{aligned}
\left\|\mathscr{B}_{N} g^{N}-f\right\|_{H} & =\left\|\sum_{n=1}^{N} \frac{1}{\sigma_{n}} a_{n} f_{n}-\sum_{n=1}^{\infty}\left(f, f_{n}\right)_{H} f_{n}\right\|_{H} \\
& =\left\|\sum_{n=1}^{N} \frac{a_{n}-\left(g, g_{n}\right)_{K}}{\sigma_{n}} f_{n}-\sum_{n=N+1}^{\infty}\left(f, f_{n}\right)_{H} f_{n}\right\|_{H} \\
& =\sqrt{\sum_{n=1}^{N}\left(\frac{a_{n}-\left(g, g_{n}\right)_{K}}{\sigma_{n}}\right)^{2}+\sum_{n=N+1}^{\infty}\left(f, f_{n}\right)_{H}^{2}} .
\end{aligned}
$$

The first term is an error we make when not choosing $a_{n}=\left(g, g_{n}\right)_{K}$. Since $\left\{g_{n}\right\}_{n-1}^{\infty}$ is an orthonormal set, with respect to the scalar product in $K$, this is how the optimal set of parameters $\left\{a_{n}\right\}_{n-1}^{N}$ should be chosen to minimize the residual in the $K$ norm. But we chose to minimize it with respect to the standard norm in order to keep the noise variance low, see Sec. II B. The second term of Eq. (28) is the residual caused by projecting $g$ onto the subspace spanned by $\left\{g_{n}\right\}_{n=1}^{N}$.

\section{EXPERIMENTS}

We have made our experiments on a brass block using a focused transducer for airborne ultrasound. The transducer was designed by Hans W. Persson, Lund Institute of Technology, Sweden, for the investigation presented in Ref. 6 and is acoustically adapted for air. It has a radius of curvature of $45 \mathrm{~mm}$, a cross-section radius of $9.5 \mathrm{~mm}$, and a center frequency of $1.0 \mathrm{MHz}$ which makes the wavelength $\lambda=0.34$ $\mathrm{mm}$.

In order to measure $g(p)$, the transducer was placed just above the edge of a brass block and moved over the edge 


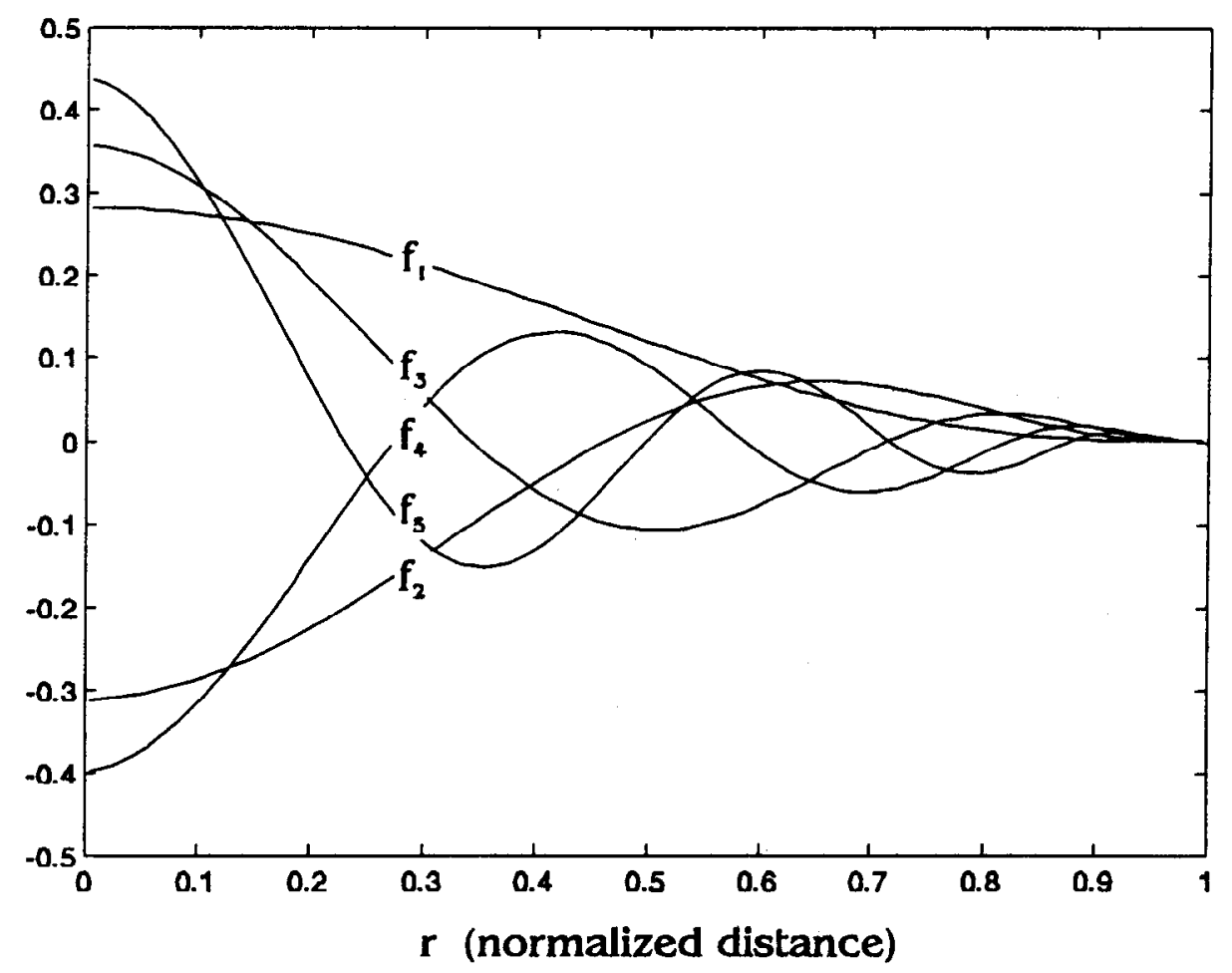

FIG. 6. The first 5 basis functions for Hilbert space $H$, the single point echoes.

using a coordinate table. To increase the SNR, an averaging of 100 echoes at position $p$ was done. However, these echoes cannot be averaged in the usual way because they all contain a small random time shift. This time shift is caused by small heterogeneities in the medium (temperature gradients and air turbulence in our case). An investigation performed by us show that there are small random time shifts in the size range of 20-30 ns in the echoes. The same problems have been reported in Ref. 18. Averaging the echoes in the normal way will both distort the shape and the arrival time of the echo. Instead, an averaging process described in Ref. 19 was used. This process estimates all the time shifts between the 100 echoes, interpolates the echoes so that they are aligned in time, and then averages them. An average of these echoes was then stored in the computer.

The sampling frequency was $20 \mathrm{MHz}$ and we took 1024 samples which makes the sweep length $51.2 \mu \mathrm{s}$. This time is sufficient for the echo to disappear almost completely. Between each measurement of a single echo, we moved the transducer $12.5 \mu \mathrm{m}$. The brass block was placed perpendicular to the transducer's movement. Using the definition of the focus width as the distance between the two first intensity minima in the focal plane, ${ }^{20}$ the focus width $d_{\text {width }}=1.8 \mathrm{~mm}$. After the measurements, we found that the echo was approximately zero when the brass block was further away than 1.25 $\mathrm{mm}$ from the axis of symmetry. Noting that this is more than the distance to first intensity minima, which is $d_{\text {width }} / 2=0.9$ mim, we neylect the contribution of points further away. We normalize this $1.25-\mathrm{mm}$ distance to $p=1$ in our calculations.

\section{RESULTS}

For the chosen weight functions described in Sec. I, the SVD was calculated. Since the Euclidean norm is not used, a modification of the standard SVD was made to numerically calculate the singular system, see Appendix B. The integral operator $\mathscr{f}$ was digitized into 100 points in $r$ and 200 in $p$, i.e., $\Delta_{r}=\Delta_{p}=0.01$. The first 5 singular functions $f_{n} \in H$ and $g_{n} \in K$ are plotted in Figs. 6 and 7, respectively.

The inversion of Eq. (5) was then done by using the spectral cut-off method. By trial-and-error, we found a choice of the regularization parameter $N$, i.e., the number of terms in the sum in Eq. (18), to be 5. The inversion of the half-plane echoes $g(p, t)$ was then performed, i.e., one inversion for each value of $t$. The resulting single point echo $f(r, t)$ is plotted as a function of time in Fig. 8 for 3 different values of $r$.

Note that the amplitude is normalized to enhance the differences in signal shape. Also note that points away from the axis of symmetry yield echoes that start earlier and have a longer duration. This is caused by the fact that these points will have a distribution of ultrasonic path lengths that include both shorter and longer ones than the focal point that is equidistant to all points on the surface of the transducer.

The intensity distribution in the focal plane of a focused transducer can be calculated analytically.,21 The Fourier transform $H(\bar{r}, \omega)$ of the spatial impulse response in the focal plane is found in Ref. 2 to be

$$
H(r, \omega)=-\frac{a^{2}}{R^{2}} e^{j \omega\left(r^{2} / 2 R c+R / c\right)} \frac{J_{1}(\omega a r / R c)}{\omega a r / R c},
$$




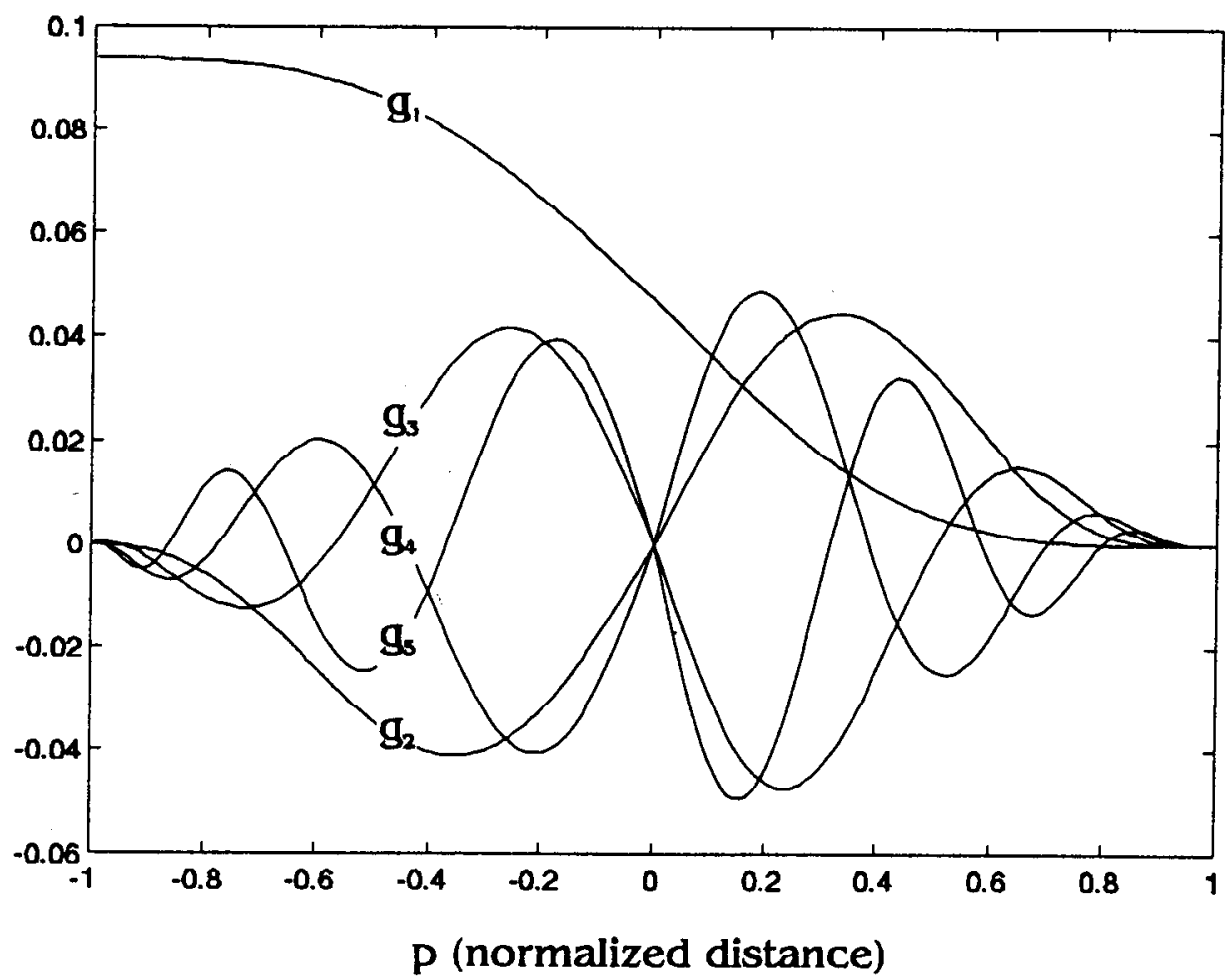

FIG. 7. The first 5 basis functions for Hilbert space $K$, the half-plane echoes.

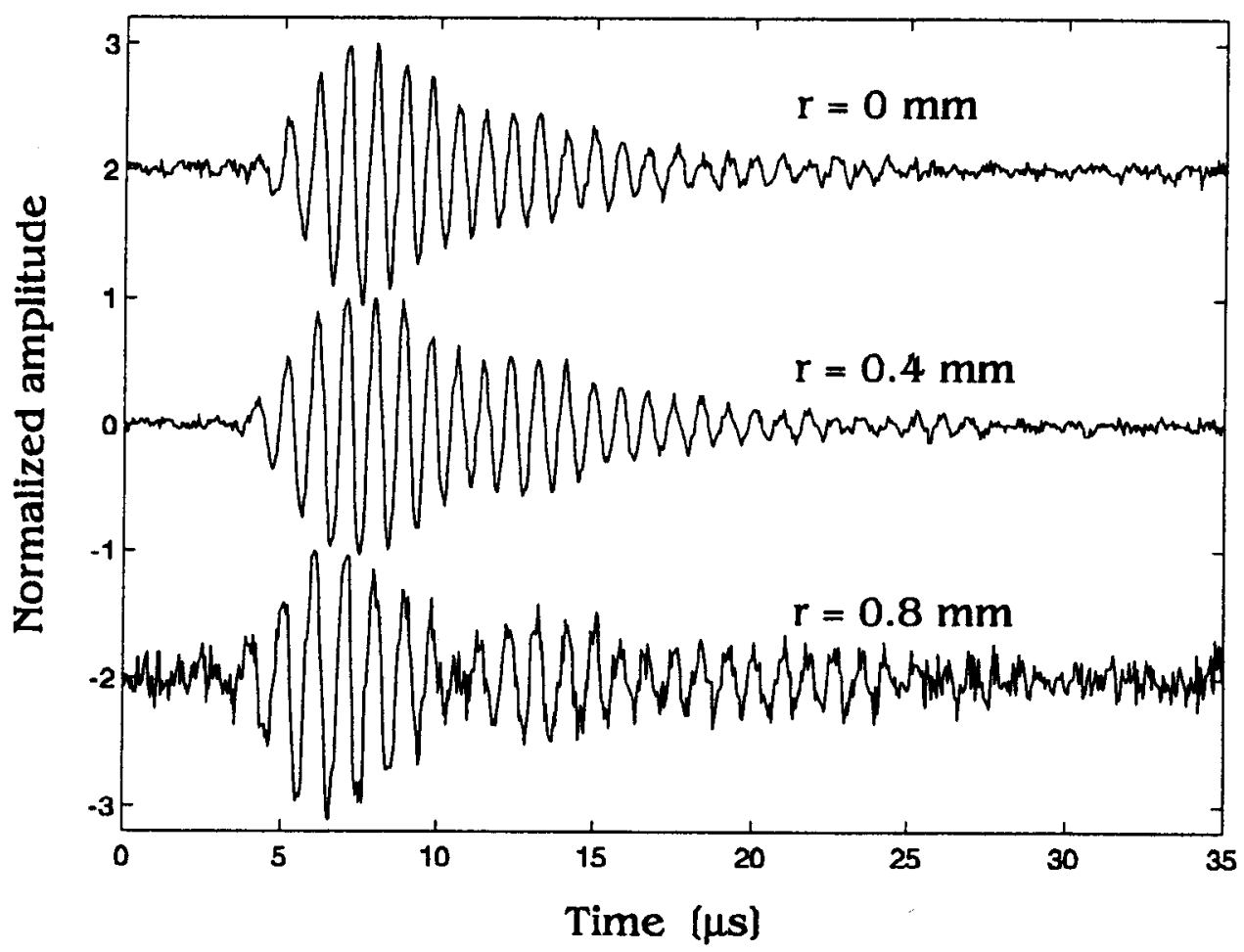

FIG. 8. Point echoes at different radial distances. 


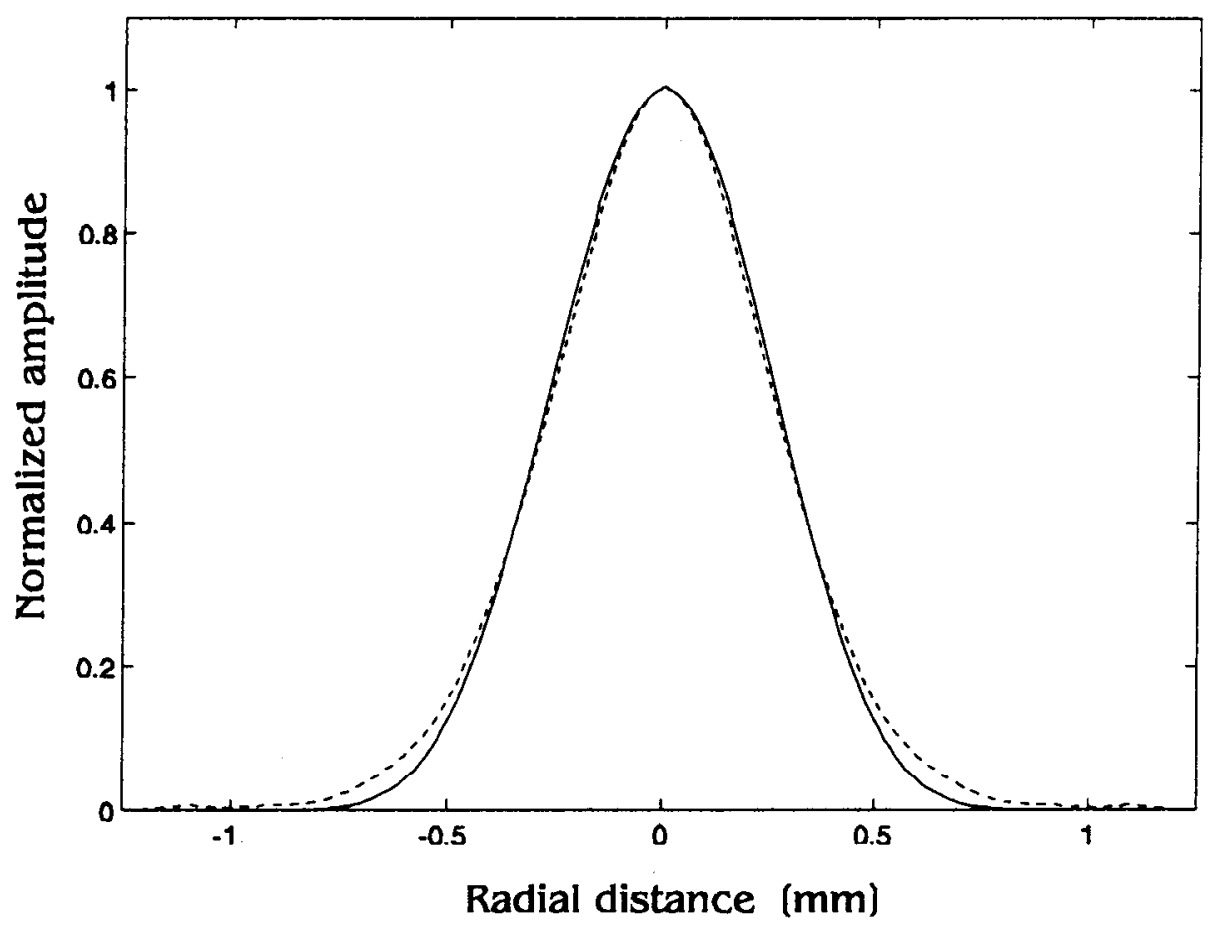

FIG. 9. Reflected energy from field point echoes: calculated (solid) and theoretical (dashed),

where $J_{1}(\cdot)$ is the first-order Bessel function of the first kind. Under the assumption that the field point intensity is proportional to $\left|H\left(r, \omega_{0}\right)\right|^{2}$, with $\omega_{0}$ being the center frequency, and using the reciprocity property, ${ }^{11}$ the reflected energy for a narrow-band pulse will be

$$
I(r) \sim\left|H\left(r, \omega_{0}\right)\right|^{4} \sim\left|\frac{J_{1}\left(\omega_{0} a r / R c\right)}{\omega_{0} a r / R c}\right|^{4} .
$$

In Fig. 9, the reflected energy of the calculated point echoes is compared to Eq. (30).

As can be seen, these two curves show a similar shape and the width of the focus corresponds well with the calculated value in Sec. III.

\section{SUMMARY}

In this paper we have dealt with the echo from a pointlike reflector, the single point echo. Knowing this quantity, a model of the echo from an reflector of arbitrary shape can be made. Assuming that the superposition principle ${ }^{4}$ is valid, the echo can be considered as the sum of all the echoes from points that make up the surface. The single point echo for an ultrasonic transducer can be measured directly but there are some practical problems attached to it. The reflector has to be very small to be considered a pointlike reflector which results in a weak echo, i.e., low SNR. If the size is increased on the other hand, there will be spatial smoothing.

Our solution to this was to use an indirect method. The echoes from sliding half-planes are measured and by using a tomographic way of reasoning, the single-point echoes can be estimated from these measurements. An operator . to mapping the single point echoes from the weighted Hilbert space
$H$ to the half-plane echoes in the weighted Hilbert space $K$ was defined. The reason why a weight function was used is that the a priori information of the echoes should be used. These weight functions were determined heuristicly from methods used in tomography. ${ }^{16}$ The inverse of the operator fo is not continuous which will result in a fatal amplification of data error such as measurement noise. Instead, we created a family of regularized inverses $\mathscr{B}_{\alpha}$ which can be made arbitrarily close to $\mathscr{A}^{-1}$ as the regularization parameter $\alpha \rightarrow \infty$. Since the resulting data error increases for increasing $\alpha$, this leads to a trade-off between approximation error and data error.

The regularization family used was the spectral cut-off method. By doing a singular value decomposition (SVD) of the operator $A$ with respect to the norms in $H$ and $K$, we can create an orthonormal base for the Hilbert spaces. By projecting the measurements to an $N$-dimensional subspace of $K$, spanned by the $N$ first basis functions, we can construct a regularized inverse to that is continuous. The basis functions and the resulting single-point echoes were shown for the chosen weight functions.

\section{APPENDIX A: THE INTEGRATION KERNEL}

The echo from a half-plane with perpendicular distance $p>0$, see Fig. 3, from the axis of symmetry can be written as

$$
\begin{aligned}
g(p) & =\int_{P} f(r) r d r d \theta=\int_{p}^{1} 2 \int_{0}^{\arccos (p / r)} r f(r) d \theta d r \\
& =\int_{p}^{1} 2 r \arccos \left(\frac{p}{r}\right) f(r) d r
\end{aligned}
$$


The relation $g(p)+g(-p)=g(-1)=2 g(0)$ can be used since the sum of two complementary parts of the unit circle equals the whole circle. Thus, for $p<0$,

$$
\begin{aligned}
g(p) & =2 g(0)-g(-p) \\
& =2 \int_{0}^{1} 2 r \frac{\pi}{2} f(r) d r-\int_{-p}^{1} 2 r \arccos \left(-\frac{p}{r}\right) f(r) d r \\
& =\int_{0}^{1} 2 \pi r f(r) d r+\int_{-p}^{1} 2 r\left[\arccos \left(\frac{p}{r}\right)-\pi\right] f(r) d r \\
& =\int_{0}^{-p} 2 \pi r f(r) d r+\int_{-p}^{1} 2 r \arccos \left(\frac{p}{r}\right) f(r) d r .
\end{aligned}
$$

The result from Eq. (A1) and Eq. (A2) can be combined to

$$
g(p)=\int_{0}^{1} K(p, r) f(r) d r,
$$

where the integration kernel is defined as

$$
K(p, r)=\left\{\begin{array}{l}
2 \pi r, \quad-1 \leqslant p<-r \\
2 r \arccos (p / r), \quad-r \leqslant p<r \\
0, \quad r \leqslant p \leqslant 1 .
\end{array}\right.
$$

\section{APPENDIX B: SVD WITH WEIGHTED NORMS}

In order to numerically calculate the SVD, we digitize the integral operator $\mathscr{A}$ described in Sec. I. We have a nonEuclidian norm since the inner products in the spaces $H$ and $K$ are defined as

$$
\left(f_{1}, f_{2}\right)_{H}=\mathbf{f}_{1}^{T} \mathbf{U} \mathbf{f}_{2}, \quad\left(g_{1}, g_{2}\right)_{K}=\mathbf{g}_{1}^{T} \mathbf{W} \mathbf{g}_{2},
$$

where $\mathbf{f}_{i}$ is a $N \times 1$ vector and $\mathbf{g}_{j}$ is a $M \times 1$ vector containing samples from the functions $f_{i}(r) \in H$ and $g_{j}(p) \in K$. The square matrices $\mathbf{U}$ and $\mathbf{W}$ are diagonal and contains samples from the weight functions $U_{4}(r)$ and $W_{4}(p)$, respectively. Since the weight functions are non-negative, $\mathbf{U}^{1 / 2}$ and $\mathbf{W}^{1 / 2}$ exist. Now we make a change of coordinates:

$$
\boldsymbol{\phi}=\mathbf{U}^{1 / 2} \mathbf{f}, \quad \gamma=\mathbf{W}^{1 / 2} \mathbf{g}
$$

Rewriting the mapping of $\mathbf{f}$ to $\mathbf{g}$

$$
\begin{aligned}
& \mathbf{A f}=\mathbf{g}, \quad \mathbf{A U ^ { - 1 / 2 }} \boldsymbol{\phi}=\mathbf{W}^{-1 / 2} \gamma, \\
& \mathbf{W}^{1 / 2} \mathbf{A U}^{-1 / 2} \boldsymbol{\phi}=\boldsymbol{\gamma}, \quad \mathbf{B} \boldsymbol{\phi}=\boldsymbol{\gamma} .
\end{aligned}
$$

The SVD of the matrix $B$ is

$$
\mathbf{B}=\left[\gamma_{1} \cdots \gamma_{N}\right] \mathbf{\Sigma}\left[\boldsymbol{\phi}_{1} \cdots \boldsymbol{\phi}_{M}\right]^{T}=\boldsymbol{\Gamma} \mathbf{\Sigma} \boldsymbol{\Phi}^{T},
$$

where $\mathbf{\Sigma}=\operatorname{diag}\left(\sigma_{1}, \sigma_{2}, \ldots\right)$ and $\sigma_{n}$ are the singular values of $\mathbf{B}$. This is a standard SVD, i.e., for these matrices we have

$$
\boldsymbol{\Phi}^{T} \boldsymbol{\Phi}=\mathbf{I}_{M}, \quad \Gamma^{T} \boldsymbol{\Gamma}=\mathbf{I}_{N} .
$$

These basis vectors, which are orthogonal with the normal Euclidian inner product, can now be transformed to obtain basis vectors for $H$ and $K$. Denoting the basis vectors for these spaces $\mathbf{f}_{n}$ and $\mathbf{g}_{n}$, we have from Eq. (B1)

$$
\begin{aligned}
\mathbf{F} & =\left[\begin{array}{llll}
\mathbf{f}_{1} & \mathbf{f}_{2} & \cdots & \mathbf{f}_{N}
\end{array}\right] \\
& =\left[\begin{array}{lllll}
\mathbf{U}^{-1 / 2} \boldsymbol{\phi}_{1} & \mathbf{U}^{-1 / 2} \boldsymbol{\phi}_{2} & \cdots & \mathbf{U}^{-1 / 2} \boldsymbol{\phi}_{N}
\end{array}\right]=\mathbf{U}^{-1 / 2} \boldsymbol{\Phi},
\end{aligned}
$$

$$
\begin{aligned}
\mathbf{G} & =\left[\begin{array}{llll}
\mathbf{g}_{1} & \mathbf{g}_{2} & \cdots & \mathbf{g}_{M}
\end{array}\right] \\
& =\left[\begin{array}{lllll}
\mathbf{W}^{-1 / 2} \boldsymbol{\gamma}_{1} & \mathbf{W}^{-1 / 2} \boldsymbol{\gamma}_{2} & \cdots & \mathbf{W}^{-1 / 2} \boldsymbol{\gamma}_{M}
\end{array}\right] \\
& =\mathbf{W}^{-1 / 2} \boldsymbol{\Gamma} .
\end{aligned}
$$

These matrices are orthogonal to the respective inner products in $H$ and $K$ :

$$
\begin{aligned}
\mathbf{F}^{T} \mathbf{U F} & =\left(\mathbf{U}^{-1 / 2} \boldsymbol{\Phi}\right)^{T} \mathbf{U}\left(\mathbf{U}^{-1 / 2} \boldsymbol{\Phi}\right)=\boldsymbol{\Phi}^{T} \mathbf{U}^{-1 / 2} \mathbf{U} \mathbf{U}^{-1 / 2} \boldsymbol{\Phi} \\
& =\boldsymbol{\Phi}^{T} \boldsymbol{\Phi}=\mathbf{I}_{M} \\
\mathbf{G}^{T} \mathbf{W} \mathbf{G} & =\left(\mathbf{W}^{-1 / 2} \boldsymbol{\Gamma}\right)^{T} \mathbf{W}\left(\mathbf{W}^{-1 / 2} \boldsymbol{\Gamma}\right) \\
& =\boldsymbol{\Gamma}^{T} \mathbf{W}^{-1 / 2} \mathbf{W} \mathbf{W}^{-1 / 2} \boldsymbol{\Gamma}=\boldsymbol{\Gamma}^{T} \boldsymbol{\Gamma}=\mathbf{I}_{N}
\end{aligned}
$$

What is left to show is that $\mathbf{f}_{n}$ and $\mathbf{g}_{n}$ really are the singular vectors of $\mathbf{A}$ with respect to the norms in $H$ and $K$. The adjoint operator $\mathscr{A}^{*}$ of $\mathscr{A}$ is defined as

$$
\left(f, A^{*} g\right)_{H}=(\mathscr{A f}, g)_{K} \quad \forall f \in H, \quad \forall g \in K .
$$

The adjoint operator of $\mathbf{A}$ is found from

$$
\begin{aligned}
& \mathbf{f}^{T} \mathbf{U}\left(\mathbf{A}^{*} \mathbf{g}\right)=(\mathbf{A f})^{T} \mathbf{W g}=\mathbf{f}^{T} \mathbf{A}^{T} \mathbf{W g}, \\
& \mathbf{U} \mathbf{A}^{*}=\mathbf{A}^{T} \mathbf{W} \Rightarrow \mathbf{A}^{*}=\mathbf{U}^{-1} \mathbf{A}^{T} \mathbf{W} .
\end{aligned}
$$

Using the following relationship of the singular vectors:

$$
\mathbf{B} \boldsymbol{\phi}_{n}=\sigma_{n} \gamma_{n}, \quad \mathbf{B}^{*} \boldsymbol{\gamma}_{n}=\sigma_{n} \boldsymbol{\phi}_{n}
$$

with $\mathbf{B}^{*}=\mathbf{B}^{T}$ (which is the case with Euclidian norm), we have

$$
\begin{aligned}
& \mathbf{B} \boldsymbol{\phi}_{n}=\sigma_{n} \boldsymbol{\gamma}_{n}, \\
& \mathbf{W}^{1 / 2} \mathbf{A U}^{-1 / 2} \boldsymbol{\phi}_{n}=\sigma_{n} \gamma_{n}, \\
& \mathbf{A} \mathbf{U}^{-1 / 2} \boldsymbol{\phi}_{n}=\sigma_{n} \mathbf{W}^{-1 / 2} \boldsymbol{\gamma}_{n}, \\
& \mathbf{A} \mathbf{f}_{n}=\sigma_{n} \mathbf{g}_{n}
\end{aligned}
$$

and

$$
\begin{aligned}
& \mathbf{B}^{T} \boldsymbol{\gamma}_{n}=\sigma_{n} \boldsymbol{\phi}_{n}, \\
& \mathbf{U}^{-1 / 2} \mathbf{A}^{T} \mathbf{W}^{1 / 2} \gamma_{n}=\sigma_{n} \phi_{n}, \\
& \mathbf{U}^{-1} \mathbf{A}^{T} \mathbf{W}\left(\mathbf{W}^{-1 / 2} \gamma_{n}\right)=\sigma_{n} \mathbf{U}^{-1 / 2} \boldsymbol{\phi}_{n}, \\
& \mathbf{A}^{*} \mathbf{g}_{n}=\sigma_{n} \mathbf{f}_{n} .
\end{aligned}
$$

We see that $\mathbf{f}_{n}$ and $\mathbf{g}_{n}$ are the singular vectors of $\mathbf{A}$ and that the singular values are the same as for $\mathbf{B}$. This means that the standard algorithms for SVD can be used to calculate the singular system of $\mathbf{A}$ when the norms are non-Euclidian.

\footnotetext{
${ }^{1}$ M. Arditi, F. S. Forster, and J. W. Hunt, "Transient fields of concave annular arrays," Ultrason. Imag. 3, 37-61 (1981).

${ }^{2}$ W. N. Cobb, "Frequency domain method for the prediction of the ultrasonic field patterns of pulsed, focused radiators," J. Acoust. Soc. Am. 75, 72-79 (1984).

${ }^{3} \mathrm{~A}$. Penttinen and M. Luukkala, "The impulse response and pressure nearfield of a curved ultrasonic radiator," J. Phys. D: Appl. Phys. 9, 15471557 (1976).

${ }^{4}$ A. Lhémery, "Impulse-response method to predict echo-responses from
} 
targets of complex geometry. Part 1," J. Acoust. Soc. Am. 90, 2799-2807 (1991).

${ }^{5}$ J. F. Havlice and J. C. Taenzer, "Medical ultrasonic imaging: An overview of principles and instrumentation," Proc. IEEE 67, 620-641 (1979).

${ }^{6}$ N. Sundström, P. O. Börjesson, N.-G. Holmer, L. Olsson, and H. W. Persson, "Registration of surface structures using airborne focused ultrasound," Ultrasound Med. Biol. 17, 513-518 (1991).

${ }^{7}$ G. C. Carter, Ed., "Special issue on time delay estimation," IEEE Trans. Acoust. Speech Signal Process. 29 (3, Pt. 2) (1981).

${ }^{8}$ G. C. Carter (Ed), Coherence and Time Delay Estimation (IEEE, New York, 1993).

${ }^{9}$ P. O. Börjesson, N.-G. Holmer, K. Lindström, and G. Salomonsson, "Notes on ultrasound echoes from angular surfaces," Technical report TR-172, Electrical Engineering Department, University of Lund (1982).

${ }^{10} \mathrm{~J}$. A. Jensen, "A model for the propagation and scattering of ultrasound in tissue," J. Acoust. Soc. Am. 89, 182-190 (1991).

${ }^{11} \mathrm{~J}$. P. Weight and A. J. Hayman, "Observations of the propagation of very short ultrasonic pulses and their reflection by small targets," J. Acoust. Soc. Am. 63, 396-404 (1978).

${ }^{12} \mathrm{~A}$. Grennberg and M. Sandell, "Experimental determination of the ultrasonic echo from a pointlike reflector using a tomographic approach," in Proceedings of the IEEE 1992 Ultrasonic Symposium, Tucson, 1992 (IEEE, New York, 1992), pp. 639-642.
${ }^{13}$ S. Deans, The Radon Transform and Some of its Applications (Wiley, New York, 1983).

${ }^{14} \mathrm{~F}$. Natterer, The Mathematics of Computerized Tomography (Wiley, New York, 1986).

${ }^{15} \mathrm{R}$. Colton and R. Kress, Inverse Acoustic and Electromagnetic Scattering Theory (Springer-Verlag, Berlin, 1992).

${ }^{16} \mathrm{~A}$. K. Louis, "Orthogonal function series expansions and the null space of the Radon transform," SIAM J. Math. Anal. 15, 621-633 (1984).

${ }^{17}$ M. Sandell, "Spatial decomposition of ultrasonic echoes," Licentiate thesis 1994:05L, Luleå University of Technology (1994).

${ }^{18}$ J. D. Fox, B. T. Khuri-Yakub, and G. S. Kino, "High-frequency acoustic wave measurements in air," in Proceedings of the IEEE 1983 Ultrasonics Symposium, Atlanta, 1983 (IEEE, New York, 1983), pp. 581-584.

${ }^{19} \mathrm{~A}$. Grennberg and M. Sandell, "Estimation of subsample time delay differences in narrow-band ultrasonic echoes using the Hilbert transform correlation," IEEE Trans. Ultrason. Ferroelec. Freq. Control 41, 588-595 (1994).

${ }^{20} \mathrm{C}$. R. Hill, "The generation and structure of acoustic fields," in Physical Principles in Medical Ultrasonics, edited by C. R. Hill (Ellis Horwood, 1986), pp. 68.92.

${ }^{21}$ G. S. Kino, Acoustics Waves (Prentice-Hall, Englewood Cliffs, NJ, 1987). 